\title{
A CHARACTERIZATION OF A SEMI-LOCALLY-CONNECTED PLANE CONTINUUM
}

\section{F. BURTON JONES}

In 1908 Schoenflies characterized a continuous curve (in the plane) by means of its complement. ${ }^{1}$ In view of more recent work, ${ }^{2}$ the kernel of his characterization may be stated as follows: In order that a plane, bounded, cyclic continuum be a continuous curve it is necessary and sufficient that (1) each of its complementary domains be simple and (2) the collection of its complementary domains be contracting. In his paper on semi-locally-connected sets, ${ }^{3} \mathrm{G}$. T. Whyburn pointed out many similarities between semi-locally-connected sets and continuous curves. In particular (it is an immediate consequence of one of his theorems $s^{4}$ ) every complementary domain of a plane, bounded, cyclic semi-locally-connected continuum is simple. But since such a continuum need not be a continuous curve, the collection of its complementary domains need not be contracting. It is the purpose of this paper to point out what characteristic property this collection of complementary domains does possess: namely, the collection contains no folded subcollection. This property, in conjunction with (1) above, characterizes a plane, bounded, cyclic semi-locally-connected continuum. In fact it is shown that a plane, bounded continuum, whether cyclic or not, is semi-locally-connected if and only if its complement is non-folded. These theorems should prove useful when constructing examples of semi-locally-connected continua which also have certain other properties.

Definitions and notation. Let space be a simple closed surface (that is, a 2 -sphere) and let $S$ denote the set of all points of space. A circular region of $S$ whose radius is $\epsilon$ and whose center is $A$ will be denoted by $U(A, \epsilon)$. If $M$ is a closed subset of $S$, each component of $S-M$

Presented to the Society, April 26, 1946; received by the editors July 5, 1946.

${ }^{1}$ A. Schoenflies, Die Entwickelung der Lehre von den Punktmannigfaltigkeiten, Leipzig, 1908, p. 237.

${ }^{2}$ R. L. Moore, Concerning the common boundary of two domains, Fund. Math. vol. 6 (1924) pp. 201-213, Theorem 9.

G. T. Whyburn, Analytic topology, Amer. Math. Soc. Colloquium Publications, vol. 28, New York, 1942, Theorem 4.4, p. 113.

${ }^{3}$ G. T. Whyburn, Semi-locally-connected sets, Amer. J. Math. vol. 61 (1939) pp. 733-749.

${ }^{4}$ Loc. cit. footnote 3, p. 749. Cf. R. L. Wilder, Sets which satisfy certain avoidability conditions, Casopis pro Pestovane Mathematicky a Fysiky vol. 67 (1938) pp. 185-198, Theorem 8a, p. 196. 
is a complementary domain of $M$. A complementary domain of a simple closed curve is called a simple domain. A connected set $M$ is said to be semi-locally-connected provided that if $P$ is a point of $M$ lying in an open subset $U$ of $M$, there exists an open subset $V$ of $M$ such that $U \supset V \supset P$ and $M-V$ is the sum of a finite number of connected sets. $^{3}$ If $A$ and $B$ are distinct points of a set $M, M$ is said to be $a p o$ syndetic at $A$ with respect to $B$ provided there exists an open subset of $M$ which contains $A$ and belongs to a connected and relatively closed subset of $M$ lying in $M-B$. If a set $M$ is aposyndetic at a point $A$ with respect to each point of $M-A, M$ is said to be aposyndetic at $A$. If a set $M$ is aposyndetic at each of its points, then $M$ is said to be aposyndetic. ${ }^{5}$

The following lemmas may be easily established with the help of the literature.

LEMMA 0 . In order that a continuum $M$ in $S$ be semi-locally-connected, it is necessary and sufficient that $M$ be aposyndetic.

LEMMA 1. If $T$ is a subcontinuum of an aposyndetic continuum $M$ of $S$, and $P$ is a point of $M-T$, then $M-P$ contains a continuum $K$ containing $T$ such that $M-K$ has no limit point in $T$.

Lemma 2. If $A$ and $B$ are distinct points of the continuum $M$ of $S$, and $M$ is not aposyndetic at $A$ with respect to $B$, then $M$ plus any finite number of its complementary simple domains is not aposyndetic at $A$ with respect to $B$.

Definition. A countably infinite collection $D_{1}, D_{2}, D_{3}, \cdots$ of mutually exclusive simple domains in $S$ is said to be folded provided there exist sets $T_{1}, T_{2}, T_{3}, \cdots$ and a point $A$ of $S$ such that (1) for each $i$, $T_{i}$ is a spanning arc-segment (open arc) lying in $D_{i}$ and separating $D_{i}$ in to two components $C_{i}$ and $E_{i}$, (2) $T_{1}, T_{2}, T_{3}, \cdots$ converges to a subset $T$ of $S-A$, and (3) for each number $\epsilon>0$, there exists an integer $n$, so that both $C_{i}$ and $E_{i}$ intersect $U(A, \epsilon)$ when $i>n$.

THEOREM 1. In order that a nondegenerate set $M$ of $S$ be a semi-locallyconnected, cyclic continuum it is necessary and sufficient that the components of $S-M$ form a collection of mutually exclusive simple domains containing no folded subcollection.

${ }^{5}$ F. B. Jones, Aposyndetic continua and certain boundary problems, Amer. J. Math. vol. 63 (1941), pp. 545-553. For the definition of other terms and phrases the reader is referred to R. L. Moore's Foundations of point set theory and G. T. Whyburn's Analytic topology, vols. 13 and 28 respectively, of the Amer. Math. Soc. Colloquium Publications, New York. 
Proof. Suppose that $M$ is a semi-locally-connected, cyclic continuum (nondegenerate). Every component of $S-M$ is a simple domain. ${ }^{4}$ Now suppose that $G$, the collection of all the components of $S-M$, contains a folded subcollection $D_{1}, D_{2}, D_{3}, \cdots$. There exists a point $A$ and, for each positive integer $i$, there exists a spanning arc-segment $T_{i}$ lying in $D_{i}$ and separating $D_{i}$ into two components $C_{i}$ and $E_{i}$ such that (1) $T_{1}, T_{2}, T_{3}, \cdots$ converges to a subset $T$ of $S-A$, but (2) if $\epsilon$ is a positive number, there exists an integer $n$, so that for $i>n, U(A, \epsilon) \cdot C_{i} \neq 0$ and $U(A, \epsilon) \cdot E_{i} \neq 0$. It is clear that $T$ is a continuum lying in $M$. By Lemma $1, M-A$ contains a continuum $K$ containing $T$ such that $M-K$ has no limit point in $T$. Let $\epsilon$ be a positive number such that $K \cdot \bar{U}(A, \epsilon)=0$. There exist an arc $L$ and an integer $n$ such that (1) the end points of $T_{n}$ belong to $K$, (2) $T_{n} \cdot U(A, \epsilon)=0$ and (3) $L$ lies in $U(A, \epsilon)$ and contains points of both $C_{n}$ and $E_{n}$. Let $H$ denote an arc of $L$ irreducible from $\bar{C}_{n}$ to $\bar{E}_{n}$; let $J$ denote the boundary of $D_{n}$; and let $Q$ denote $S-\left(D_{n}+J\right)$. Both $H$ and $K$ lie in $J+Q$. Furthermore the end points of $H$ separate the end points of $T_{n}$ from each other on $J$. But since $K$ contains the end points of $T_{n}, K$ must intersect $H$. This is a contradiction and establishes the necessity of the condition stated in Theorem 1.

Now suppose that $G$ is a collection of mutually exclusive simple domains which contains no folded subcollection. Let $M$ denote the closed set $S-G^{*} \cdot{ }^{6}$ If $M$ were not connected, then there would exist a simple closed curve $J$ such that $M$ would not intersect $J$ but would intersect each complementary domain of $J$. Consequently neither complementary domain of $J$ would be a subset of the element of $G$ containing $J$. Since this is impossible, $M$ must be connected. Suppose that $M$ is not aposyndetic at a point $A$. Then there exists a point $B$ such that if $W$ is a subcontinuum of $M-B, A$ is a limit point of $M-W$. Let $U_{1}, U_{2}, U_{3}, \cdots$ denote a monotone descending sequence of circular regions centering on $A$ and converging to $A$. Let $V_{1}, V_{2}$, $V_{3}, \cdots$ denote a monotone descending sequence of circular regions centering on $B$ and converging to $B$ such that $\bar{U}_{1} \cdot \bar{V}_{1}=0$. Although $M \cdot\left(S-V_{1}\right)$ contains $M \cdot \bar{U}_{1}$, no component of $M \cdot\left(S-V_{1}\right)$ contains $M \cdot U_{1}$. Hence $M \cdot\left(S-V_{1}\right)$ is the sum of two mutually exclusive closed sets $M_{1}$ and $N_{1}$ each of which contains a point of $U_{1}$. There exists an arc in $U_{1}$ irreducible from $M_{1}$ to $N_{1}$; let $T_{1}$ denote this arc less its end points. The arc-segment $T_{1}$ lies in an element $D_{1}$ of $G$ and separates it into two domains $C_{1}$ and $E_{1}$ each of which contains a point of $V_{1}$ since no arc in the boundary of $D_{1}$ lies in $S-V_{1}$ and contains

- $G^{*}$ denotes the sum of the elements of $G$. 
both end points of $T_{1}$. Using Lemma 2 it is easy to see that no component of $\left(M+D_{1}\right) \cdot\left(S-V_{2}\right)$ contains $M \cdot U_{2}$. Hence $\left(M+D_{1}\right) \cdot\left(S-V_{2}\right)$ is the sum of two mutually exclusive closed sets $M_{2}$ and $N_{2}$ each of which contains a point of $U_{2}$. There exists an arc in $U_{2}$ irreducible from $M_{2}$ to $N_{2}$; let $T_{2}$ denote this arc less its end points. The arcsegment $T_{2}$ lies in an element $D_{2}$ of $G$ and separates it into two domains $C_{2}$ and $E_{2}$ each of which contains a point of $V_{\mathbf{2}}$. Again no component of $\left(M+D_{1}+D_{2}\right) \cdot\left(S-V_{3}\right)$ contains $M \cdot U_{3}$. Hence $\left(M+D_{1}+D_{2}\right) \cdot\left(S-V_{3}\right)$ is the sum of two mutually exclusive closed sets $M_{3}$ and $N_{3}$ each of which contains a point of $U_{3}$. There exists an arc in $U_{3}$ irreducible from $M_{3}$ to $N_{3}$; let $T_{3}$ denote this arc less its end points. The arc-segment $T_{3}$ lies in an element $D_{3}$ of $G$ and separates it in to two domains $C_{3}$ and $E_{3}$ each of which contains a point of $V_{3}$. Continue this process indefinitely. Since the sequence $T_{1}, T_{2}, T_{3}, \ldots$ converges to $A$ and $V_{1}, V_{2}, V_{3}, \cdots$ converges to $B, G$ contains a folded subcollection $D_{1}, D_{2}, D_{3}, \cdots$ contrary to hypothesis. Hence $M$ is aposyndetic at every one of its points and is, by Lemma 0 , semi-locally-connected. Now suppose that $P$ is a cut point of $M$. Then $M$ is the sum of two closed sets $H$ and $K$ such that $H \cdot K=P$. Let $A B$ denote an arc in $S-P$ irreducible from $H$ to $K$. Since $A B-(A+B)$ lies in an element of $G, A$ and $B$ belong to a simple closed curve lying in $M$. Hence $P$ does not separate $A$ from $B$ in $M$. Thus the assumption that $M$ contains a cut point has led to a contradiction, and the sufficiency of the condition stated in Theorem 1 is established.

COROLlary 1. In order that a plane, bounded, cyclic continuum $M$ be semi-locally-connected (or aposyndetic) it is necessary and sufficient that (1) each complementary domain of $M$ be simple and (2) the collection of complementary domains of $M$ contain no folded subcollection.

Remarks. Theorem 1 serves in a way as a characterization of a true cyclic element of a semi-locally-connected continuum (in a 2 -sphere). In order to characterize a semi-locally-connected continuum (whether cyclic or not) by means of its complement, it is necessary to extend the notion of foldedness to open sets in general.

Definition. If $D$ is an open set, $D$ is said to be folded provided that there exist in $D$ mutually exclusive sets $X_{1}, X_{2}, X_{3}, \cdots$ such that (1) for each $i(i=1,2,3, \cdots), X_{i}$ is the sum of two arc-segments $T_{i 1}$ and $T_{i 2}$ having their end points in the boundary of $D$, (2) for each $i, T_{i 1}$ crosses $T_{i 2}$, (3) the sequence $T_{11}, T_{21}, T_{31}, \cdots$ converges to a subset $T$ of $S-D$, and (4) the sequence of end points of $T_{32}, T_{22}$, 
$T_{32}, \cdots$ converges to a point of $S-T$. If the open set $D$ is not folded, $D$ is said to be non-folded. ${ }^{7}$

THEOREM 2. In order that the continuum $M$ of $S$ be semi-locally-connected (or aposyndetic) it is necessary and sufficient that $S-M$ be nonfolded.

Proof. The condition is sufficient. For suppose, on the contrary, that $M$ is not aposyndetic at a point $A$ of $M$ with respect to a point $B$ of $M$. Let $U_{1}, U_{2}, U_{3}, \cdots$ denote a monotone descending sequence of circular regions centered on and converging to $A$, and let $V_{1}, V_{2}$, $V_{3}, \cdots$ denote a monotone descending sequence of circular regions centered on and converging to $B$ such that $\bar{U}_{1} \cdot \bar{V}_{1}=0$. Now the component of $M \cdot\left(S-V_{1}\right)$ which contains $A$ is not open (relative to $M$ ) at $A$. Hence the boundary of $U_{1}$ contains an arc-segment $T_{11}$ whose end points, $X_{1}$ and $Y_{1}$, lie in different components of $M \cdot\left(S-V_{1}\right)$ such that $M \cdot T_{11}=0$. There exists a simple closed curve which separates $X_{1}$ from $Y_{1}$ and contains no point of $M \cdot\left(S-V_{1}\right)$; in fact, there exists a simple closed curve $J_{1}$ which separates $X_{1}$ from $Y_{1}$ and contains no point of $M \cdot\left(S-V_{1}\right)$ such that $J_{1} \cdot T_{11}$ is connected. In $J_{1}$ plus the boundary of $V_{1}$ there exists an arc-segment $T_{12}$ which crosses $T_{11}$, lies in $S-V_{1}$, and has only its end points in $M$. Obviously these end points lie in the boundary of $V_{1}$. Let $F_{2}$ denote the boundary of $U_{2}$ and let $K_{2}$ denote the sum of the components of $F_{2}-F_{2} \cdot M$ which intersect $T_{12}$. Then $K_{2}$ is the sum of a finite number of arc-segments. Now since the component of $M \cdot\left(S-V_{2}\right)$ which contains $A$ is not open (relative to $M$ ) at $A$ and $T_{11}+T_{12}+K_{2}$ has only a finite number of limit points in $M$, the component of $\left(M+T_{11}+T_{12}+K_{2}\right) \cdot\left(S-V_{2}\right)$ which contains $A$ is not relatively open at $A$. Hence $F_{2}$ contains an arc-segment $T_{21}$ whose end points, $X_{2}$ and $Y_{2}$, lie in $M$ and in different components of $\left(M+T_{11}+T_{12}+K_{2}\right) \cdot\left(S-V_{2}\right)$ such that $\left(M+T_{11}+T_{12}+K_{2}\right) \cdot T_{21}=0$. There exists a simple closed curve $J_{2}$ which separates $X_{2}$ from $Y_{2}$, and contains no point of $\left(M+T_{11}+T_{12}+K_{2}\right) \cdot\left(S-V_{2}\right)$ such that $J_{2} \cdot T_{21}$ is connected. In $J_{2}$ plus the boundary of $V_{2}$ there exists an arcsegment $T_{22}$ which crosses $T_{21}$, lies in $S-V_{2}$, and has only its end points in $M$. These end points lie in the boundary of $V_{2}$. Furthermore, $\left(T_{11}+T_{12}\right) \cdot\left(T_{21}+T_{22}\right)=0$. This process may be continued indefinitely. Since $T_{11}, T_{21}, T_{31}, \cdots$ converges to $A$ and the sequence of end points of $T_{12}, T_{22}, T_{32}, \cdots$ converges to $B, S-M$ is folded, contrary to hypothesis. Hence the condition is sufficient.

${ }_{7}$ The reader should observe that no simple domain is folded and that if $G$ is a collection of mutually exclusive simple domains, $G^{*}$ is folded if and only if $G$ contains a folded subcollection. 
The condition is also necessary. For suppose, on the contrary, that $S-M$ is folded. Then $S-M$ contains an infinite sequence of pairs of arc-segments $T_{11}, T_{12}, T_{21}, T_{22}, \ldots$ such that (1) for each $i, T_{i 1}$ and $T_{i 2}$ span $M$, (2) for each $i, T_{i 1}$ crosses $T_{i 2}$, (3) for each $i$ and $j$ $(i \neq j),\left(T_{i 1}+T_{i 2}\right) \cdot\left(T_{j 1}+T_{j 2}\right)=0,(4)$ the sequential limiting set $T$ of $T_{11}, T_{21}, T_{31}, \ldots$ is a subset of $M$, and (5) the sequence of end points of $T_{12}, T_{22}, T_{32}, \cdots$ converges to a point $B$ not belonging to $T$. By Lemma $1, M-B$ contains a continuum $K$ and an open subset $U$ of $M$ such that $K \supset U \supset T$. Let $V$ denote a circular region containing $B$ such that $K \cdot \bar{V}=0$. There exists an integer $i$, such that $T_{i 1}$ has its end points in $K$ but $\bar{V} \cdot T_{i 1}=0$, and such that $T_{i 2}$ has its end points in $V$. But since $T_{i 1}$ crosses $T_{i 2}$, this is impossible.

Corollary 2. In order that a plane, bounded continuum be semilocally-connected (or aposyndetic) it is necessary and sufficient that its complement be non-folded.

THEOREM 3. In order that the boundary of a simply connected domain $D$ be a continuous curve it is necessary and sufficient that $D$ be non-folded.

Proof. If $D$ is non-folded, $S-D$ is an aposyndetic continuum $M$. Hence the boundary of $D$ is a continuous curve. ${ }^{8}$ On the other hand, if $\beta$, the boundary of $D$, is a continuous curve, then $\beta$ is aposyndetic, and $S-\beta$ is non-folded. Hence $D$ is non-folded.

The University of Texas

8 See footnotes 3 and 5. Cf. R. L. Wilder, Property $S_{n}$, Amer. J. Math. vol. 61 (1939) pp. 823-832, p. 832 in particular. 